\title{
The Effects of Aging and Solvent-Vapor Treatments on the Viscoelastic Properties of Star-Shaped Styrene-Butadiene Radial Block Copolymers
}

\author{
Toshikazu Takigawa, Yasuhiko OHta, and Toshiro Masuda \\ Research Center for Medical Polymers and Biomaterials, \\ Kyoto University, Sakyo-ku, Kyoto 606, Japan
}

(Received September 20, 1989)

\begin{abstract}
The purpose of the present study is to see the effect of aging and solvent-vapor treatment on the viscoelasticity of star-shaped radial block copolymers. The pahse morphology determined by the viscoelasticity was compared with that of as-cast films on which we reported in the previous paper. By aging for 6 months, the submolecularly heterogeneous phase (SMHP) was segregated into each polystyrene and polybutadiene domain. The formation of pinched-in lamellae of polybutadine was accelerated by aging. According to the solvent-vapor treatment for aged films, the polystyrene and polybutadiene domains resultant from the SMHP by aging, were re-mixed. However, the formation of the pinched-in lamellae was further enhanced.

KEY WORDS Styrene / Butadiene / Radial Block Copolymer / Viscoelasticity / Aging / Solvent-Vapor Treatment /
\end{abstract}

Effects of aging on polymeric materials have been widely studied ${ }^{1-6}$ by many researchers for both scientific and industrial interests. The studies suggest that the viscoelastic and mechanical properties of the polymeric materials are strongly affected by the temperature and term of aging. However, studieds ${ }^{7}$ on the aging effect of the block copolymers are still few in spite of their importance. In the previous paper, ${ }^{8}$ we reported the relationship between the morphology, and the viscoelastic and mechanical properties of star-shaped styrene(S)-butadiene(B) radial block copolymers. S-B radial block copolymer films cast from mixed solvent of tetrahydrofuran (THF) and methylethylketone (MEK), showed a unique morphology of submolecularly heterogeneous phase (SMHP), which was found gray by transmission electron microscopy (TEM). Dynamic viscoelastic properties of the films were strongly affected by the morphology. The SMHP showed the glass transition temperature $\left(T_{\mathrm{g}}\right)$ at about $80^{\circ} \mathrm{C}$, which is lower than the $T_{\mathrm{g}}$ $\left(=100^{\circ} \mathrm{C}\right)$ of pure polystyrene (PS). On the other hand, the film specimens cast from cyclohexane (Cy) solution showed a characteristic $T_{\mathrm{g}}$ at about $60^{\circ} \mathrm{C}$, being assigned to the glass transition of pinched-in lamellae. The SMHP and pinched-in lamellae were considered not to be in equilibrium state but in transient morphology, which were frozen in the course of film preparation. Consequently, it is very interesting how the morphology of the films changes by aging. Another interest is to investigate the effect of solvent absorption on the morphological change in the films, because the absorbed solvent might make the chain mobility high and accelerate the structural changes. In this study, we examined the effects of the aging and solvent vapor treatments on the dynamic viscoelastic properties of films of the star-shaped S-B block copolymers.

\section{EXPERIMENTAL}

The star-shaped S-B radial block copoly- 
Table I. Number- and weight-average molecular weights and ratios for radial block copolymers, length and composition of the branch ( $\mathrm{S}$, weight percentage of styrene) measured in number-average molecular weight and coupling ratio $(\mathrm{P}$, average number of branches per molecule)

\begin{tabular}{|c|c|c|c|c|c|c|c|c|}
\hline \multirow{2}{*}{ Sample } & \multicolumn{3}{|c|}{ Radial block copolymer } & \multicolumn{3}{|c|}{ Branch $M_{n} \times 10^{-4}$} & \multirow{2}{*}{$\mathrm{S} / \%$} & \multirow{2}{*}{$\mathrm{P}$} \\
\hline & $M_{n} \times 10^{-4}$ & $M_{w} / M_{n}$ & $M_{w} \times 10^{-4}$ & SB & PS & PB & & \\
\hline SBH1 & 25.7 & 1.13 & 29.0 & 5.64 & 4.63 & $1.01^{\mathrm{a}}$ & 82.1 & 4.6 \\
\hline BSH1 & 31.6 & 1.18 & 37.2 & 5.93 & $4.23^{\mathrm{a}}$ & 1.70 & 71.3 & 5.3 \\
\hline
\end{tabular}

a Calculated value, a difference between $M_{n}$ of SB and PS (or PB).

mers employed in this study were prepared by anionic polymerization technique. The detailed procedure was described in our previous paper. ${ }^{8}$ Table I shows the sample name and molecular characteristics of the copolymer. $\mathrm{SBH} 1$ is a radial block copolymer composed of inner polybutadiene (PB) and outer PS blocks, and BSH1 has the reverse molecular structure of SBH1. The film specimens for dynamic viscoelastic measurements were prepared by solution-cast method, and $\mathrm{Cy}$ and THF/MEK ( $9: 1$ by weight) mixture were used as casting solvents. Aging was performed at room temperature for about 6 months in a dessicator under reduced pressure. For solvent-vapor treatments, ethylbenzene (EtBz) and cyclohexanone (CyCO) were used as solvents. $\mathrm{EtBz}$ is a good solvent for both PS and PB blocks, and CyCO is reported ${ }^{9}$ as a good solvent for PS and less good for PB. The solvent-vapor treatments were carried out by keeping aged-films in a dessicator saturated by vapor of a solvent for 2 weeks at room temperature, and then drying them in a vacuum oven at $40^{\circ} \mathrm{C}$ for 2 weeks. Dynamic viscoelastic properties of the specimens were carried out using Rheovibron DDV-IIC (Orientec Co.) at a frequency of $110 \mathrm{~Hz}$.

\section{RESULTS}

\section{Effects of Aging}

Figure 1 shows the temperature dispersion curves of dynamic Young's modulus $\left(E^{\prime}\right)$ and

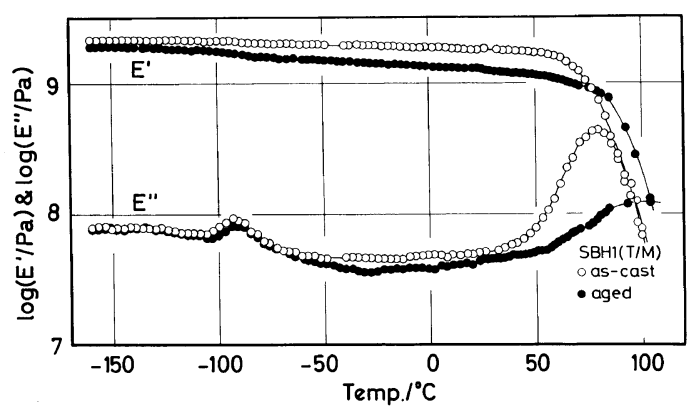

Figure 1. Temperature dispersions of dynamic Young's modulus $\left(E^{\prime}\right)$ and loss modulus $\left(E^{\prime \prime}\right)$ of as-cast $(O)$ and aged (O) $\mathrm{T} / \mathrm{M}$ films of $\mathrm{SBH}$.

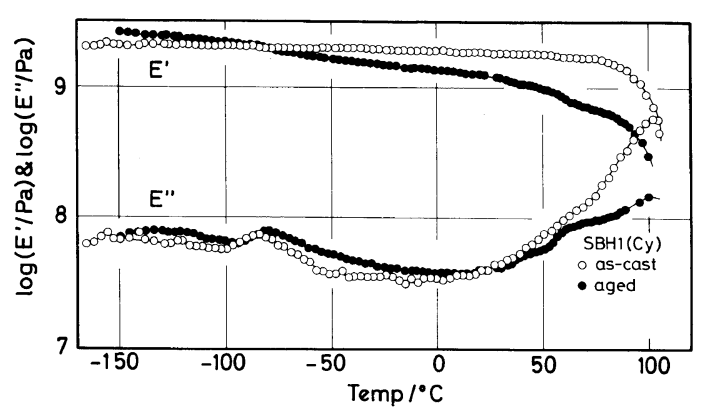

Figure 2. Temperature dispersions of dynamic Young's modulus $\left(E^{\prime}\right)$ and loss modulus $\left(E^{\prime \prime}\right)$ of as-cast $(O)$ and aged (O) Cy films of SBH1.

loss modulus $\left(E^{\prime \prime}\right)$ of the as-cast and aged films of SBH1 cast from $\mathrm{T} / \mathrm{M}$ solutions (T/M films). By aging, peaks of $E^{\prime \prime}$ located at 80 and $-95^{\circ} \mathrm{C}$ shifted to 100 and $-90^{\circ} \mathrm{C}$, respectively.

In Figure 2, a similar comparison between the as-cast and aged films of SBH1 cast from Cy solutions (Cy films) are shown. From this figure, it is observed that a broad peak of $E^{\prime \prime}$ 
in the high temperature range $\left(50^{\circ} \mathrm{C}<T<\right.$ $100^{\circ} \mathrm{C}$ ) seems to split into a peak and a shoulder during aging. The peak temperature is, however, unchanged and at $100^{\circ} \mathrm{C}$. The shoulder of $E^{\prime \prime}$ is observed at $60^{\circ} \mathrm{C}$. A small peak at lower temperatures $\left(-100^{\circ} \mathrm{C}<T<\right.$ $-60^{\circ} \mathrm{C}$ ) slightly shifted to the high temperature side by aging.

Figure 3 shows similar plots for the as-cast and aged $\mathrm{Cy}$ films of $\mathrm{BSH} 1$ having a reverse molecular structure of SBH1. A shoulder is observed on the dispersion curve of $E^{\prime \prime}$ for each film, and the corresponding temperature $\left(60^{\circ} \mathrm{C}\right)$ is not changed. A peak seen in as-cast film at $100^{\circ} \mathrm{C}$ might also remain unchanged in position by aging, though the peak is not clearly observed for aged specimen.

\section{Effects of Solvent-Vapor Treatments}

Figure 4 shows the temperature dispersion curves of $E^{\prime}$ and $E^{\prime \prime}$ for the aged and EtBz-vapor treated $\mathrm{T} / \mathrm{M}$ films of SBH1. The vapor-treated film showed two peaks of $E^{\prime \prime}$ located at 70 and $-95^{\circ} \mathrm{C}$, respectively. Both peaks clearly moved to lower temperatures in comparison with the corresponding ones for aged film. Similar curves for the aged and EtBz-vapor-treated Cy films of $\mathrm{SBH} 1$ are shown in Figure 5. A peak and a shoulder observed in the high temperature region in the dispersion curve of $E^{\prime \prime}$ for the aged film changed to a broad single peak after the solvent treatment. The peak temperature is, however, unchanged. For the peak located at low temperature side, the shift from -80 to $-90^{\circ} \mathrm{C}$ is also observed.

Figure 6 shows the temperature dispersion curves of $E^{\prime}$ and $E^{\prime \prime}$ for the aged and CyCO-vapor-treated T/M films of SBH1. A peak of $E^{\prime \prime}$ seen at $100^{\circ} \mathrm{C}$ for the aged film shifted to $60^{\circ} \mathrm{C}$ by the solvent treatment. For the peak in the lower temperature region a small shift to the lower temperatures is also observed. Figure 8 shows the similar plots for the aged and CyCO-vapor-treated $\mathrm{Cy}$ films of $\mathrm{BSH} 1$ which has the reverse molecular

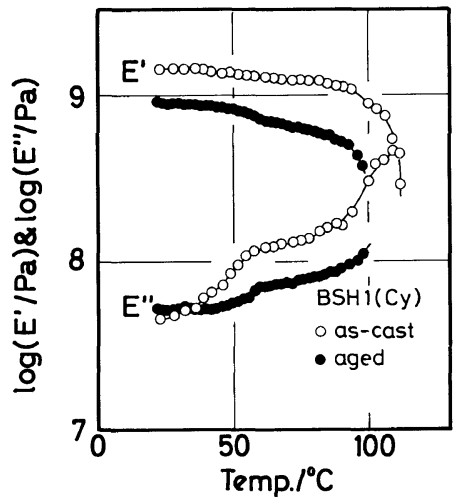

Figure 3. Temperature dispersions of dynamic Young's modulus $\left(E^{\prime}\right)$ and loss modulus $\left(E^{\prime \prime}\right)$ of as-cast $(O)$ and aged (O) Cy films of BSH1.

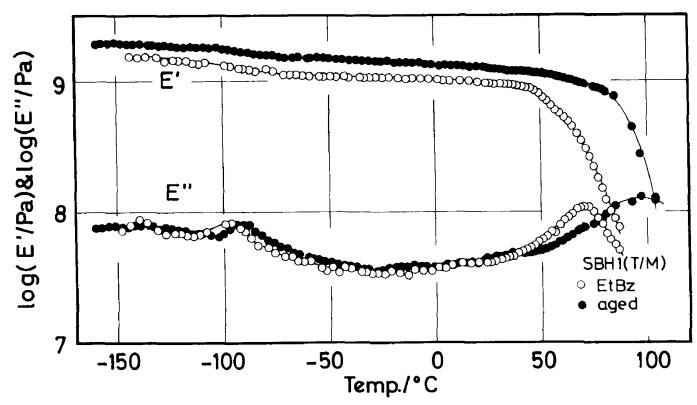

Figure 4. Temperature dispersions of dynamic Young's modulus $\left(E^{\prime}\right)$ and loss modulus $\left(E^{\prime \prime}\right)$ of aged $(O)$ and EtBz-vapor treated (O) T/M films of SBH1.

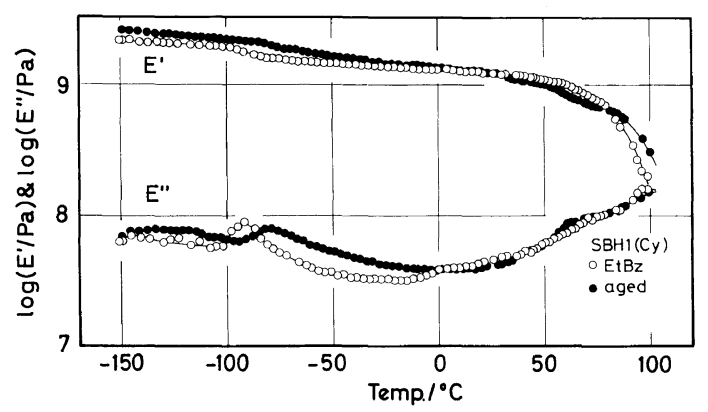

Figure 5. Temperature dispersions of dynamic Young's modulus $\left(E^{\prime}\right)$ and loss modulus $\left(E^{\prime \prime}\right)$ of aged $(O)$ and EtBz-vapor treated (O) Cy films of SBH1.

structure of SBH1. The peak position of $E^{\prime \prime}$ remains unchanged, and the temperature corresponding to the shoulder also remains at the same temperature by the treatment. 


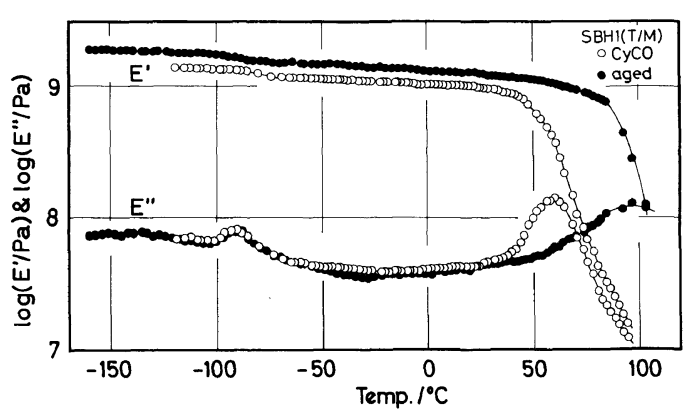

Figure 6. Temperature dispersions of dynamic Young's modulus $\left(E^{\prime}\right)$ and loss modulus $\left(E^{\prime \prime}\right)$ of aged $(O)$ and CyCO-vapor treated (O) T/M films of SBH1.

\section{DISCUSSION}

We demonstrated in our previous paper ${ }^{8}$ that temperature dispersion curves of $E^{\prime}$ and $E^{\prime \prime}$ for the radial block copolymers in a temperature range of $-150-100^{\circ} \mathrm{C}$ showed combinations of five glass transition characteristics of several types of domains made up by PS, PB, or both chains. They are glass transitions of:

(1) pure PS domains observed in the temperature range of $100-110^{\circ} \mathrm{C}$.

(2) pure PB domains observed in a wide range of temperature of -100 to $-65^{\circ} \mathrm{C}$, depending on the morphology.

(3) interphase region between PS and PB domains, observed continuously between $T_{\mathrm{g}}$ 's of PB and PS, and resulting in a gradual decrease in $E^{\prime}$ curve and a shallow minimum of $E^{\prime \prime}$ in the temperature range.

(4) PS domains including pinched-in lamellae of PB, observed around $60^{\circ} \mathrm{C}$.

(5) phases where star-shaped copolymers are molecularly dispersed but submolecularly heterogeneous (SMHP), observed around $80^{\circ} \mathrm{C}$.

It was also shown ${ }^{8}$ that the morphology of the star-shaped radial block copolymers was strongly dependent on casting solvent and could be classified into several categories on the basis of the viscoelasticity measurements and TEM observations. The $T / M$ film of SBH1 showed a morphology with disordered cylinders of PB and the Cy film, a morphology with the disordered lamellae. The Cy film of BSH1 showed ordered lamellar morphology. The morphology of disordered lamellae, ordered lamellae and disordered cylinders was in non-equilibrium. On the other hand, the ordered cyclinders of PB in SBH1 and BSH1 appeared thermodynamically stable. Hence, it might be of great interest to investigate morphological changes by aging of the solvent cast samples.

During the solvent-vapor treatments, morphological change occurs in a swollen state and the resultant morphologies may remain even after rapid drying. The morphological change is due to the thermodynamic state of the system and the enhanced mobility of polymer chains in swollen state.

\section{Residual Solvent in Solvent-Vapor-Treated Films after Drying}

The amount of solvent absorbed by the film could not be measured quantitatively. However, all the films treated by $\mathrm{EtBz}_{-}$and CyCO-vapor were solid and kept their shapes at their equilibrium absorptions. A residual solvent in dried films, if any, must exert an influence upon the viscoelastic properties. It is specially important here to discuss the residual solvent in vapor-treated films, because EtBz and $\mathrm{CyCO}$ are less volatile than the casting solvents, THF/MEK and Cy. In order to check the effect of residual solvent on viscoelasticity of film specimens, CyCO-vapor treatment was carried out for PS homopolymer. The film specimens for radial block copolymers used in this study have continuous phases of PS regardless of the morphology. ${ }^{8}$ The $T_{\mathrm{g}}$ of PS for both homopolymer and PS phase of the radial block copolymers must be similarly affected by the solvent, if the solvent remains after drying. The CyCO-vapor treated film of PS homopolymer, however, showed no downward shift of $T_{\mathrm{g}}$. Moreover, Cy films of the 


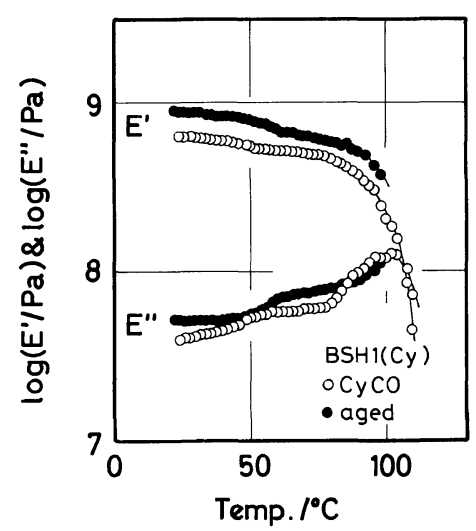

Figure 7. Temperature dispersions of dynamic Young's modulus $\left(E^{\prime}\right)$ and loss modulus $\left(E^{\prime \prime}\right)$ of aged $(O)$ and CyCO-vapor treated $(O)$ Cy films of BSH1.

radial block copolymers also showed no shift of $T_{\mathrm{g}}$ of PS domains by $\mathrm{CyCO}$-vapor treatment as seen from Figure 7 as an example. These results definitely imply that residual solvent is too little, if any, to affect the relaxation phenomena in CyCO-vapor-treated specimens. This is, of course, applicable for $\mathrm{EtBz}$-vapor -treated film because EtBz is also good for PS. As seen from Figures 4 and 6, the CyCO-and EtBZ-vapor-treated T/M films showed a downward shift of $T_{\mathrm{g}}$ of PS. The shift must not be attributed to the residual solvent but to another reason as discussed below. We need not worry about residual solvent in PB domains, because the mobility of PB chains is extremely high at $40^{\circ} \mathrm{C}$ in a vacuum oven. Consequently, the effect of plasticization of the residual solvent on the $T_{\mathrm{g}}$ is also negligible.

\section{$T / M$ Film of $S B H 1$}

A large peak of $E^{\prime \prime}$ of the as-cast film located at $80^{\circ} \mathrm{C}$ in Figure 1 was assigned ${ }^{8}$ to the glass transition of (5) SMHP. This peak temperature moves to $100^{\circ} \mathrm{C}$ by aging, as can be seen from Figure 1. The temperature corresponds to the $T_{\mathrm{g}}$ of (1), PS pure domains. The as-cast $\mathrm{T} / \mathrm{M}$ film showed disordered cylindrical morphology which contained the SMHP. The SMHP is a special structure where radial block copolymer chains are molecularly dispersed but the parts of PS and PB within a molecule are segregated. The morphology is not thermodynamically stable and the region is observed gray by TEM. $^{8}$ In the course of aging, small PB domains located at the inner part of a molecule aggregate to form bigger domains. We could not determine size by TEM, because the resultant morphology was still gray, suggesting that the size of the resultant PB domains is smaller than the resolution of TEM. In contrast with the TEM observation, the morphological change reflects sensitively the temperature dispersion curve of $E^{\prime \prime}$. Two peaks corresponding to respective PS and PB domains are distinguishable by the viscoelasticity measurements. These are the higher $T_{\mathrm{g}}$ of (1), and the lower $T_{\mathrm{g}}$ of (2), pure PB domains. This also implies a morphological change from disordered cylinders to ordered cylinders of PB by aging, because the segregation process in SMHP which lowers free energy of the system and is a stage toward a stable morphology of ordered cylinders.

The peak of $E^{\prime \prime}$ located at a lower temperature $\left(-95\right.$ and $\left.-90^{\circ} \mathrm{C}\right)$ shown in Figure 1 was assigned to $T_{\mathrm{g}}$ of (2) for the as-cast and aged films. PB pure domains in the as-cast film do not change by aging and still contribute to the $T_{\mathrm{g}}$ of (2) in the aged film. The relaxation intensity of this transition must increase by aging to some extent. However, the increment is not clearly observed in Figure 1. The relaxation intensity of the glass transition at $100^{\circ} \mathrm{C}$ for aged film is expected to be lower than that at $80^{\circ} \mathrm{C}$ for as-cast film, because the SMHP transition will be split into those of pure PS and PB domains. The $E^{\prime \prime}$ curve of the aged $\mathrm{T} / \mathbf{M}$ film in Figure 1 still shows a limited intensity around $80^{\circ} \mathrm{C}$, suggesting that the conversion of SMHP (5) to pure domains, (1) and (2), is not completed by the aging period. This might be the reason for the unchanged peak at the low temperature and the lower peak at $100^{\circ} \mathrm{C}$ than expected.

Small upward shift $\left(-95 \rightarrow-90^{\circ} \mathrm{C}\right)$ of the 
$T_{\mathrm{g}}$ of (2) by aging is observed in Figure 1. It is reported ${ }^{7}$ that the PB domains in PS matrix feel the negative pressure upon cooling before measurements because of a mismatch in the thermal expansion coefficients between PS and $\mathrm{PB}$, which causes a dilation of the PB domains at lower temperatures. This makes the $T_{\mathrm{g}}$ of (2) for the block copolymers lower than $-65^{\circ} \mathrm{C}$ for $\mathrm{PB}$ homopolymer ${ }^{8}$ with the same molecular structure. A densification of PS glassy phase may progress during aging and reduce the negative pressure ${ }^{7}$ on $\mathrm{PB}$ domains at lower temperatures.

As can be seen from Figure 4, EtBz-vaportreated T/M film of SBH1 shows a $T_{\mathrm{g}}$ at $70^{\circ} \mathrm{C}$. This can be assigned to the $T_{\mathrm{g}}$ of (5), though it is lower than the $T_{\mathrm{g}}$ of (5) observed in the as-cast film shown in Figure 1. In the aged film, as discussed before, there are PB domains originating from SMHP as well as those observed in the as-cast film. The former PB domains and the surrounding PS domains would be re-mixed to form the SMHP by the absorption of EtBz and be frozen during evaporation of the solvent, as SMHP is thermodynamically more stable than a segregated structure in swollen state. Similarly in Figure 6, CyCO-vapor-treated $\mathrm{T} / \mathrm{M}$ film shows a $T_{\mathrm{g}}$ at $60^{\circ} \mathrm{C}$. This also corresponds to the $T_{\mathrm{g}}$ of (5), though $T_{\mathrm{g}}$ is lower than that for $\mathrm{EtBz}$-vapor-treated film by $10^{\circ} \mathrm{C}$. The difference in $T_{\mathrm{g}}$ for SMHP among as-cast $\left(T_{\mathrm{g}}=\right.$ $\left.80^{\circ} \mathrm{C}\right)$, EtBz-treated $\left(T_{\mathrm{g}}=70^{\circ} \mathrm{C}\right)$, and $\mathrm{CyCO}$ treated $\left(T_{\mathrm{g}}=60^{\circ} \mathrm{C}\right)$ can be attributed to the difference in plasticizing efficiencies of $\mathrm{PB}$ chains; in other words, it depends on the solvent $(\mathrm{T} / \mathrm{M}, \mathrm{EtBz}$, and $\mathrm{CyCO})$ used and probably on the process of vaporization. As for the $T_{\mathrm{g}}$ of (2), a small upward shift was observed in the EtBz- and CyCO-vapor-treated films, due to the disappearance of the densification effect by a re-formation of the SMHP.

\section{Cy Film of SBHI}

The effects of aging and solvent-treatments on viscoelastic properties of SBH1 Cy films are shown in Figures 2, 5, and 7. A broad peak and a shoulder in each $E^{\prime \prime}$ curve of Figure 2 were observed in $60-100^{\circ} \mathrm{C}$. These relaxations were assigned to the superposed glass transitions of (1), (4) (PS + pinched-in PB lamellae) and (5). ${ }^{8}$ The shoulder becomes clearer by aging. The peak temperature remains unchanged. The as-cast film showed ${ }^{8}$ a "disordered lamellar morphology" which includes PS domains (1), SMHP (5) and PB lamellae partly pinched-in (4). The micro-phase separation in SMHP mentioned in the previous section occurs again here, corresponding to the morphological change from (5) to (1) and (2). The $T_{\mathrm{g}}$ of SMHP in this film is not clear from the figure but is, of course, lower than that of (1). The Cy film with the disordered lamellar morphology is not in equilibrium where it should have an ordered cylindrical one. On the way toward morphological change on aging from disordered lamellae to ordered cylinders of PB an increment of the pinched-in lamellar region (4) must be required to achieve the equilibrium state. Finally we can conclude qualitatively that the SMHP (5) is split into pure PS (1) and PB (2) domains, while the original PS domain (1) and the pinched-in PB lamellae (4) remain unchanged. As for the $T_{\mathrm{g}}$ of pure PB domains (2), a small upward shift is observed in $T_{\mathrm{g}}$, as seen in Figure 2. This is due to the densification effect of PS domains, as discussed before. The densification in PS domains will, of course, occur independently of the formation of the pinched-in lamellae of PB.

The EtBz-vapor-treated Cy film of SBH1 showed again a broad peak of $E^{\prime \prime}$ at the higher temperatures as seen in Figure 5. We can see no big difference in viscoelastic properties between the two samples in contrast with the case of Figure 4. The formation of the pinched-in PB lamellae would be favored by absorption of the solvent. The amounts of solvent absorbed by the film is small, so that mixing of PS and PB in the lamellar region does not occur because it is thermodynamically 
unfavored. However, the lamellar morphology itself is also thermodynamically unstable. The stable morphology in swollen state is the cylinders of PB. On the other hand, absorbed solvent enhances the mobility of polymer chains. This causes increased formation of pinched-in lamellae of PB which are in a transient morphology to PB cylinders. The relaxation intensity of glass transition of pinched-in lamellae (4) might be enhanced in treated film as is observed at $60^{\circ} \mathrm{C}$ in Figure 5 . A downward shift of $T_{\mathrm{g}}$ of (2) by the treatment is due to the dilation (anti-densification) generated in PS domains by the treatment as we observed for as-cast films. The corresponding result of $\mathrm{CyCO}$ treatment is not shown here because the CyCO-Treated $\mathrm{Cy}$ film showed a quite similar dispersion curve of $E^{\prime \prime}$ to that of the EtBz-treated Cy film shown in Figure 5. All discussion on EtBz treatments of Cy films is valid for $\mathrm{CyCO}$ treatments. Consequently, big differences in aging and solvent-vapor treatment behavior between $\mathrm{T} / \mathrm{M}$ and $\mathrm{Cy}$ films of the radial block copolymers originate from the morphology in the as-cast films. The phase separation proceeds earlier (at lower concentration and viscosity) in the cyclohexane solution than in the THF/MEK solution, causing the SMHP morphology to remain more in the $\mathrm{T} / \mathrm{M}$ film. The details are discussed in the previous paper. ${ }^{8}$

\section{The Cy Film of BSHI}

The Cy film of BSH1, having the reverse molecular structure of SBH1, showed no change in shape in the temperature dispersion curves of $E^{\prime}$ and $E^{\prime \prime}$ by aging, shown in Figure 3. Since the as-cast film contains only lamellar region, ${ }^{8}$ the peak and shoulder observed in the dispersion curve of $E^{\prime \prime}$ for the as-cast film are assigned to the glass transitions of (1) and (4), respectively. The $T_{\mathrm{g}} \mathrm{s}$ of (1) and (4) were unchanged by aging. By the $\mathrm{CyCO}$-vapor treatment shown in Figure 7, the shape of the dispersion curves seems almost unchanged. This is bacause the $T_{\mathrm{g}}$ of (1) and (4) are not

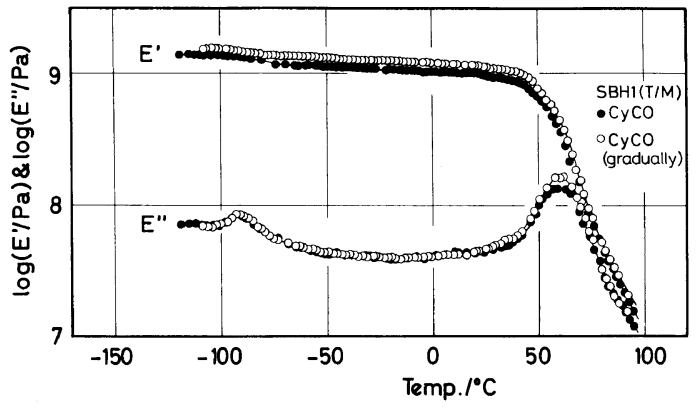

Figure 8. Temperature dispersions of dynamic Young's modulus $\left(E^{\prime}\right)$ and loss modulus $\left(E^{\prime \prime}\right)$ of the T/M films of SBH1 dried in vacuum oven (O), and gradually in the atomosphere and then in a vacuum oven $(O)$.

influenced by the CyCO-vapor treatment, as discussed in the previous section.

\section{Effects of Drying Rate on the Viscoelasticity}

It would be very significant to see the effects of the rate of film drying process on the resultant morphology as well as viscoelastic properties of the films. How the drying rate affected the viscoelastic functions of a $\mathrm{T} / \mathrm{M}$ film of SBH1 swollen by $\mathrm{CyCO}$ is seen in Figure 8. The open circles correspond to the data obtained for a film dried in vacuum after leaving it in the atomosphere for a week, and filled ones the data for the film dried under usual drying conditions described in experimental section. No difference in the shapes of the dispersion curves of $E^{\prime}$ and $E^{\prime \prime}$ can be seen for the two specimens. The peaks of the $E^{\prime \prime}$ located at 60 and $-95^{\circ} \mathrm{C}$ are commonly observed. This implies that the re-formation of the SMHP (5) occurs in the swollen state and the morphology is kept afterward in the drying process.

Temperature dispersion curves of $E^{\prime}$ and $E^{\prime \prime}$ of the T/M film of SBH1 prepared by extracting $\mathrm{CyCO}$ from the swollen film by methanol before drying are shown in Figure 9, together with those of the aged one. The figure can be compared with Figures 6 and 8. The extraction of $\mathrm{CyCO}$ by methanol in the swollen state of the film clearly prevents re-formation of SMHP 


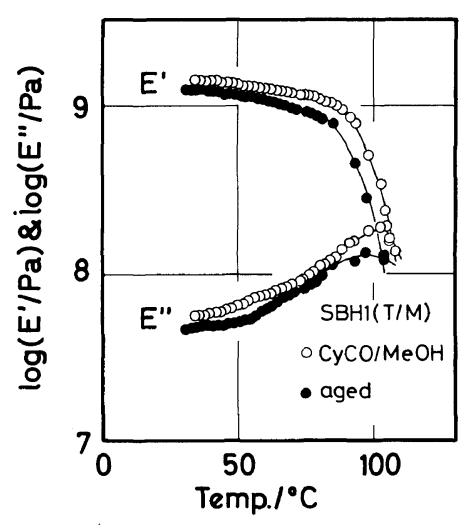

Figure 9. Temperature dispersions of dynamic Young's modulus $\left(E^{\prime}\right)$ and loss modulus $\left(E^{\prime \prime}\right)$ of the T/M films of $\mathrm{SBH} 1$. Open circles represent data for the films dried in the vacuum oven followed by extraction of $\mathrm{CyCO}$ in methanol. Filled circles show data for the aged film.

morphology. The immersion of the swollen film in methanol causes a rapid phase separation of SMHP into PS and PB domains. Phase separation may be possible in the early stage of the extraction because of a high mobility of the molecules.

\section{CONCLUSIONS}

The effects of aging and solvent-vapor treatment on the viscoelastic properties of star-shaped S-B radial block copolymers were studied and changes in morphology were examined. Comparing the results obtained in this study with those of as-cast films reported in the previous paper, the following conclusions were drawn:

1. SMHP (submolecularly heterogeneous phase) and pinched-in lamellae were nonequilibrium morphologies of S-B radial block copolymers.

2. The SMHP separates into each PS and PB domain, and the formation of the pinched-in lamellae was ehanced by aging.

3. Morphological changes can be explained by a thermodynamical transfer to a stable state of PB cylinders.

4. The PS and PB domains resultant from SMHP are re-mixed by our solvent-vapor treatments for aged specimens.

5. The formation of pinched-in lamellae is enhanced by the solvent-vapor treatments because of an increased mobility of polymer chains.

\section{REFERENCES}

1. L. C. E. Struik, "Physical Aging in Amorphous Polymers and Other Materials," Elsevier Scientific Publishing Company, New York, N.Y., 1973.

2. S. Matsuoka, H. E. Bair, S. S. Brearder, H. E. Kern, and J. T. Ryan, Polym. Eng. Sci., 18, 1073 (1978).

3. C. Bauwens-Crowet and J.-C. Bauwens, Polymer, 23, 1599 (1982).

4. R.-J. Roe and G. M. Miliman, Polym. Eng. Sci., 23, 318 (1983).

5. L. M. Carapellucci, A. F. Yee, and R. P. Nimmer, Polym. Eng. Sci., 27, 773 (1987).

6. Y. P. Chen and J. J. Aklonis, Polym. Eng. Sci., 27, 1275 (1987).

7. F. S. Bates, R. E. Cohen, and A. S. Argon, Macromolecules, 16, 1108 (1983).

8. T. Takigawa, Y. Ohta, S. Ichikawa, T. Kojima, A. Tanaka, and T. Masuda, Polym. J., 20, 293 (1988).

9. A. Bearmish, R. A. Goldberg, and D. J. Hourston, Polymer, 18, 49 (1977).

10. D. S. Herman, D. J. Kinning, E. L. Thomas, and L. J. Fetters, Macromolecules, 20, 2940 (1987). 